\title{
Targeted therapy in the treatment of uterine serous carcinoma
}

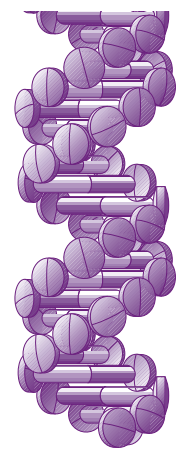

\author{
"Out of ... aggressive histologic types, uterine serous cancers are the \\ most common and most aggressive variant. If these cancers recur, they \\ are less responsive to chemotherapy and usually fatal."
}

Keywords: HER2 • PIK3CA • mTOR • targeted therapy • uterine serous carcinoma

Endometrial cancer is the most common of gynecologic malignancies in the US with nearly 52,630 diagnoses and 8590 deaths in 2014 [1]. Endometrial cancers are thought of in two broad categories; type I and type II. Type I tumors make up the majority of all new diagnoses and are estrogen and progesterone receptor positive. These hormonally driven tumors are typically diagnosed at an early stage, are endometrioid in histology and are highly curable. Type II endometrial cancers are characterized by more aggressive histotypes including serous, clear cell, mucinous, squamous, transitional cell, mesonephric and undifferentiated. Type II endometrial cancers are responsible for the majority of deaths from endometrial cancer [2]. Unlike type I endometrial cancers, these carcinomas are generally not hormonally driven or responsive to hormonal treatments making potentially available treatments more limited. Out of these aggressive histologic types, uterine serous cancers (USC) are the most common and most aggressive variant. If these cancers recur, they are less responsive to chemotherapy and usually fatal. Initial treatment for USC comprises surgical staging usually followed by cytotoxic chemotherapy with or without radiation therapy. Despite aggressive treatment, uterine serous carcinomas carry a far worse prognosis (5-year sur- vival of $30 \% \pm 9)$ and recurrence rate with $50-80 \%$ of patients developing recurrence after initial treatment [2-4].

The aggressive nature of uterine serous carcinoma has led to extensive study of molecular characteristics to better understand the tumor biology and what drives their proliferation. Data suggest a number of molecular alterations are found in USC with mutations affecting genomic stability and cell cycle regulation including the deletion or mutation of TP53 tumor suppressor gene among a number of other mediators, chromatin remodeling genes involved in the NuRD pathway and proliferation signaling mediated through both amplification and/or mutations in HER2/neu and PIK3CA [5]. While a number of different molecular targets have been identified in USC, the HER2/neu regulated PIK3CA/AKT/mTOR pathway remains the most promising target for therapy at this time.

Uterine serous carcinoma harboring HER2/neu gene amplification has been identified in up to $35 \%$ of newly diagnosed USC patients [6]. While amplification has been associated with overall worse prognosis, it may provide an ideal target for therapy. The Gynecologic Oncology Group assessed the use of single-agent trastuzumab in patients with endometrial cancer and

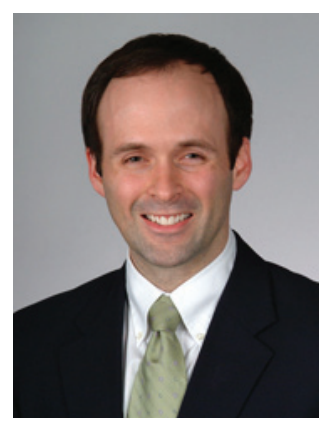

Carlton L Schwab Department of Obstetrics, Gynecology \& Reproductive Sciences, Yale School of Medicine, Rm 305 LSOG, 333 Cedar St, PO Box 208063, New Haven, CT 06520, USA

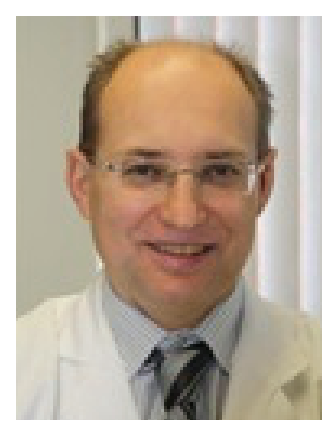

Alessandro D Santin Author for correspondence: Department of Obstetrics, Gynecology \& Reproductive Sciences, Yale School of Medicine, Rm 305 LSOG, 333 Cedar St, PO Box 208063, New Haven, CT 06520, USA alessandro.santin@yale.edu 
found that trastuzumab did not improve outcome [7]. Ongoing clinical trials have been designed to assess the use of trastuzumab in a more homogenous group of patients with HER2/neu amplified uterine serous carcinoma and in combination with chemotherapy (NCT01367002). While trials are ongoing, new data have emerged regarding the targeting of the HER2/neu pathway in USC. Including use of antibody cytotoxic drug conjugates, small tyrosine kinase inhibitors, and dual HER2/neu pathway targeted therapy.

"Initial treatment for uterine serous cancer comprises surgical staging usually followed by cytotoxic chemotherapy with or without radiation therapy. Despite aggressive treatment, uterine serous carcinomas carry a far worse prognosis ... and recurrence rate...

Drug-antibody conjugates are attractive for use in HER2/neu targeted therapy. The drug-antibody conjugate, trastuzumab ematansine (T-DM1), has shown promise in preclinical studies with improved responses seen in HER2/neu amplified USC xenografts when compared with trastuzumab alone $(\mathrm{p}<0.0001)[8]$. The improved efficacy seen in xenografts may have important implications for ongoing trials and future drug design. There is, however, some evidence to suggest that some uterine serous carcinomas maybe somewhat resistant to HER2/neu antibody-based therapy. Clinical data from breast cancer suggest that truncated p95HER2 may confer resistance to trastuzumab-based therapy [9]. These same truncated variants of HER 2 are also expressed in uterine serous carcinoma [10]. Truncation of the HER2 receptors extracellular component may make the binding of antibody more difficult or fleeting. Furthermore, previous studies have shown that HER2/neu-amplified USC can shed portions of the receptor which may act as a relative sink for circulating antibody [11]. Finally, mutations in the PIK3CA gene, a major determinant of trastuzumab resistance in breast cancer patients [12], are commonly detected in USC [5]. Overcoming these potential mechanisms of resistance maybe possible using small tyrosine kinase inhibitors or by targeting downstream signaling molecules.

Preclinical data using small irreversible tyrosine kinase inhibitors in HER2/neu-amplified USC have been published $[13,14]$. Small tyrosine kinase inhibitors act by binding within ATP pocket of the intracellular domain of the ErbB family of receptors. They target cysteine residues, which are conserved among the ErbB receptors. Data from these studies suggest that both afatinib and neratinib can inhibit signaling through the HER2/neu pathway, which leads to cell cycle arrest, decreasing proliferation and ultimately leading to improved survival in animal models. A number of clinical trials are ongoing or have been completed which were designed to show the potential efficacy of these once daily, oral, targeted agents in both ErbB mutated and amplified cancers of the lung, breast, GI tract, head and neck and urothelial tumors. In fact, data regarding the use of afatinib in non-small-cell lung cancer changed the paradigm by which EGFR-mutated non-small-cell lung cancers are treated [15]. Given new preclinical data in HER2/neuamplified USC, similar trials should be considered in HER2/neu-amplified uterine serous carcinoma.

Whole-exome sequencing data from 57 uterine serous carcinomas suggest that alterations to the HER2/neu-regulated PIK3CA/AKT/mTOR pathway are found in up to $80 \%$ of uterine serous tumors [5]. Another strategy for targeted therapy includes PI3Kand mTOR-targeted drugs. The use of a number of different compounds that target molecules within this pathway have been tested against HER2/neu-amplified uterine serous carcinoma in the preclinical setting and show significant activity including AZD8055 (target mTORC1/2), GDC-0980 (target Class I PI3K and mTORC 1/2) and GDC-0032 (target PIK3CA) [16-18]. Noting the frequency of amplification and mutation in the PI3K gene in uterine serous carcinoma inhibitors of this molecule are of particular interest. Current clinical trials using GDC 0032 (Taselisib) are seeking to determine the tolerability of PI3K inhibitors in the treatment of recurrent solid tumors. Results of these trials are eagerly anticipated.

\section{"Targeted therapy against driver pathways will continue to expand into the clinical setting and will likely be the foundation for the treatment of all cancers including uterine serous carcinoma in the future.}

Finally, data from breast cancer research have suggested that dual targeting of the HER2/neu pathway may improve outcomes in treating HER 2/neu-amplified tumors [19]. Similarly a study recently published in Clinical Cancer Research suggests that dual targeting of HER2/neu with Trastuzumab and lapatinib led to better inhibition of tumor growth in HER2/neuamplified USC xenografts [20]. These data suggest that there may be improvement in responses if a dual targeting strategy is implemented in the treatment of uterine serous carcinoma.

Uterine serous cancer is an aggressive variant of endometrial cancer, which currently has limited treatments and is nearly uniformly fatal once a patient develops recurrence. USC which overexpresses HER2/neu are 
known for being particularly aggressive but represent a subset of uterine cancer for which targeted therapy may potentially be highly effective. With USC recurrence rates as high as $50 \%$ for patients with stage I disease, it is imperative that new treatments and treatment strategies be explored. While the promise of the aforementioned targeted strategies in the preclinical setting is encouraging their clinical feasibility, toxicity and efficacy need to be explored in appropriately designed clinical trials. Targeted therapy against driver pathways will continue to expand into the clinical setting and will likely be the foundation for the treatment of all cancers including uterine serous carcinoma in the future.

\section{References}

1 American Cancer Society. Cancer Facts and Figures 2014, American Cancer Society, Atlanta, GA, USA 2014. www.cancer.org

2 Hamilton CA, Cheung MK, Osann Ket al. Uterine papillary serous and clear cell carcinomas predict for poorer survival compared with grade 3 endometrioid corpus cancers. $\mathrm{Br}$. J. Cancer 94(5), 642-646 (2006).

3 Hendrickson M, Ross J, Eifel P, Martinez A, Kempson R. Uterine papillary serous carcinoma: a highly malignant form of endometrial adenocarcinoma. Am. J. Surg. Pathol. 6(2), 93-108 (1982).

4 El-Sahwi KS, Schwartz PE, Santin AD. Development of targeted therapy in uterine serous carcinoma, a biologically aggressive variant of endometrial cancer. Expert Rev. Anticancer Ther. 12(1), 41-49 (2012).

5 Zhao S, Choi M, Overton JD et al. Landscape of somatic single-nucleotide and copy-number mutations in uterine serous carcinoma. Proc. Natl Acad. Sci. USA 110 (8), 2916-2921 (2013).

6 Buza N, English DP, Santin AD, Hui P. Toward standard HER 2 testing of endometrial serous carcinoma: 4-year experience at a large academic center and recommendations for clinical practice. Mod. Pathol. 26(12), 1605-1612 (2013).

7 Fleming GF, Sill MW, Darcy KM et al. Phase II trial of trastuzumab in women with advanced or recurrent, HER2positive endometrial carcinoma: a Gynecologic Oncology Group study. Gynecol. Oncol. 116(1), 15-20 (2010).

8 English DP, Bellone S, Schwab CL et al. T-DM1, a novel antibody-drug conjugate, is highly effective against primary HER 2 overexpressing uterine serous carcinoma in vitro and in vivo. Cancer Med. 3(5), 1256-1265 (2014).

9 Scaltriti M, Rojo F, Ocana A et al. Expression of p95HER2, a truncated form of the HER2 receptor, and response to antiHER2 therapies in breast cancer. J. Natl Cancer Instit. 99(8), 628-638 (2007).

10 Growden WB, Digloria C, Borger D et al. HER2 overexpressing high grade endometrial cancer expresses high levels of p95HER2 variant. Reprod. Sci. 21, 94A (2014).

11 Todeschini P, Cocco E, Bellone $S$ et al. Her2/neu extracellular domain shedding in uterine serous carcinoma:

\section{Financial \& competing interests disclosure}

This work was supported in part by grants from NIH R01 CA154460-01A1, the Honorable Tina Brozman Foundation, the Deborah Bunn Alley Ovarian Cancer Research Foundation, and the Guido Berlucchi Research Foundation to AD Santin. This investigation was also supported by NIH Research Grant CA-16359 from the $\mathrm{NCl}$. The authors have no other relevant affiliations or financial involvement with any organization or entity with a financial interest in or financial conflict with the subject matter or materials discussed in the manuscript apart from those disclosed.

No writing assistance was utilized in the production of this manuscript.

implications for immunotherapy with trastuzumab. $B r$. J. Cancer 105(8), 1176-1182 (2011).

12 Rexer BN, Arteaga CL. Intrinsic and acquired resistance to HER2-targeted therapies in HER2 gene-amplified breast cancer: mechanisms and clinical implications. Crit. Rev. Oncogen. 17(1), 1-16 (2012).

13 Schwab CL, Bellone S, English DP et al. Afatinib demonstrates remarkable activity against HER2-amplified uterine serous endometrial cancer in vitro and in vivo. Br. J. Cancer 111(9), 1750-1756 (2014).

14 Schwab CL, English DP, Roque DM et al. Neratinib shows efficacy in the treatment of HER 2/neu amplified uterine serous carcinoma in vitro and in vivo. Gynecol. Oncol. 135(1), 142-148 (2014).

15 Sequist LV, Yang JC, Yamamoto N et al. Phase III study of afatinib or cisplatin plus pemetrexed in patients with metastatic lung adenocarcinoma with EGFR mutations. J. Clin. Oncol. 31(27), 3327-3334 (2013).

16 English DP, Roque DM, Carrara L et al. HER2/neu gene amplification determines the sensitivity of uterine serous carcinoma cell lines to AZD8055, a novel dual mTORC1/2 inhibitor. Gynecol. Oncol. 131(3), 753-758 (2013).

17 English DP, Bellone S, Cocco E et al. Oncogenic PIK3CA gene mutations and HER2/neu gene amplifications determine the sensitivity of uterine serous carcinoma cell lines to GDC-0980, a selective inhibitor of Class I PI3 kinase and mTOR kinase (TORC1/2). Am. J. Obstetr. Gynecol. 209(5), 465.e1-9 (2013).

18 Lopez S, Schwab CL, Cocco E et al. Taselisib, a selective inhibitor of PIK3CA, is highly effective on PIK3CAmutated and HER2/neu amplified uterine serous carcinoma in vitro and in vivo. Gynecol. Oncol. 135(2), 312-317 (2014).

19 Baselga J, Bradbury I, Eidtmann $\mathrm{H}$ et al. Lapatinib with trastuzumab for HER2-positive early breast cancer (NeoALTTO): a randomised, open-label, multicentre, Phase 3 trial. Lancet 379(9816), 633-640 (2012).

20 Groeneweg JW, Hernandez SF, Byron VF et al. Dual HER2 targeting impedes growth of HER2 gene-amplified uterine serous carcinoma xenografts. Clin. Cancer Res. $20(24), 6517-6528$ (2014). 